A C T A C H E M I C A S C A N D I N A V I C A 8 (1954) $1813-1826$

\title{
Purification of Bacitracin Polypeptides by Charcoal Chromatography and Zone Electrophoresis
}

\author{
JERKER PORATH \\ Institute of Biochemistry, University of Uppsala, Uppsala, Sweden
}

\begin{abstract}
Experimental conditions for the fractionation of polypeptides by charcoal chromatography are discussed. By combining charcoal chromatography and zone electrophoresis in cellulose columns an efficient fractionation of a commercial crude bacitracin preparation was achieved. Peptides, nucleotide(s) and amino acids were resolved as different fractions. Two new peptides similar to bacitracin A, called bacitracin $H$ and $I$, were isolated. The ultraviolet absorption curves of the peptides are compared with those of fractions reported in the literature which were obtained by counter-current distribution. A description of a simple apparatus for zone electrophoresis in vertical columns is also given.
\end{abstract}

$\mathbf{E}$ fficient purification of bacitracins, antibiotic peptides obtained from Bacillus licheniformis has been achieved by Craig and Newton and their collaborators ${ }^{1-4}$. Both groups of investigators use only counter-current extraction. In spite of the inherent flexibility of this method, a complete resolution of such complicated mixtures often requires the application of complementary procedures which depend on different physical or chemical properties. Since the mixture is composed of closely related peptides the advantage of using independent methods is evident. UV-absorption curves and other data of characterization, published by the above-mentioned authors was considered to make bacitracins particularly favorable objects for comparative studies.

In a previous report, a bacitracin preparation (purified by counter-current extraction) was shown to contain considerable amounts of impurities ${ }^{5}$. The method used (carrier displacement analysis on charcoal) was, however, inconvenient for purification of crude polypeptide mixtures where the peptide to be purified is not the main component. Moreover, under the conditions used, the yield of activity and material was not entirely satisfactory. As was recently shown in the cases of insulin and ACTH-polypeptides, severe losses may be avoided by the use of properly pretreated charcoal ${ }^{6}$.

The pretreatment of the charcoal consists of deactivation of the most active adsorption centers by means of a saturator as was suggested by Tiselius and

Acta Chem. Scand. 8 (1954) No. 10 
co-workers ${ }^{7,8}$. The saturator may be incorporated in the eluting or displacing solvents (flowing saturator). Pretreated charcoal should be used, for otherwise extensive irreversible adsorption may occur. Poor recovery always results from strong adsorption, even if a very strong displacer is used to effect the desorption. On the other hand, if charcoal is pretreated with a large quantity of a strong saturator, adsorption to a significant extent may not occur. Somewhere between these extremes, a concentration range of the saturator can presumably be found where the peptide will be only weakly adsorbed. Within, or above this concentration range, chromatography must be performed to secure a high yield of the peptide in question. Thus, suitable working conditions can be found if the recovery of the peptide is determined in a series of displacement analysis experiments using varying saturator concentrations and keeping other factors constant. When this is done it is found, as could be anticipated, that the recovery increases as the concentration of the saturator increases. At a certain concentration of the pretreating agent the peptide appears in the eluate with only slight retention or without any adsorption at all occurring. The saturater concentration at which this takes place has been called the critical saturation point of the peptide. This concentration is dependent upon a number of factors which may all be easily controlled.

In view of these facts the first full scale chromatogram should be performed with charcoal at a saturation close to the critical saturation point of the component having the strongest adsorbability. The latter can be recovered by displaoement, while the other components will be found in the eluate. By repeating this procedure an appropriate number of times using charcoal at decreasing saturations, a not too complex mixture may be resolved.

Desorption can be effected either by displacement (as assumed in the preceding discussion) or by gradient elution. Displacement analysis has the advantage of being easily reproducible and thus more suitable for group separations. The proper conditions can be determined with a few milligrams and applied on a gram scale, as is shown in this paper. According to Drake it is usually not possible to attain better separation by gradient elution ${ }^{9}$. Displacement analysis is therefore preferable in preparative charcoal chromatography.

Instead of attempting a complete separation of a complex mixture such as the crude bacitracin using charcoal chromatography alone it appears to be more efficient to use this procedure only for a group separation. When necessary, other methods can be explored for further resolution. In this work zone electrophoresis was chosen as a supplementary procedure because the electrophoretical separation presumably depends on entirely different properties of the material to be resolved. Unfortunately, only a narrow $\mathrm{pH}$ range of buffers could be employed, for bacitracin $A$ is unstable and bacitracin $F$ has a low solubility at higher $\mathrm{pH}$ values. Acetate buffer of $\mathrm{pH} 4.5$ was used throughout this investigation. Zone electrophoresis of amounts less than $1 / 2 \mathrm{gram}$ may easily be performed in throughs or in columns. Vertical columns were preferred because better defined zones yielding more satisfactory separations could be obtained. The peptide material was easily freed from buffer salts by charcoal chromatography. 


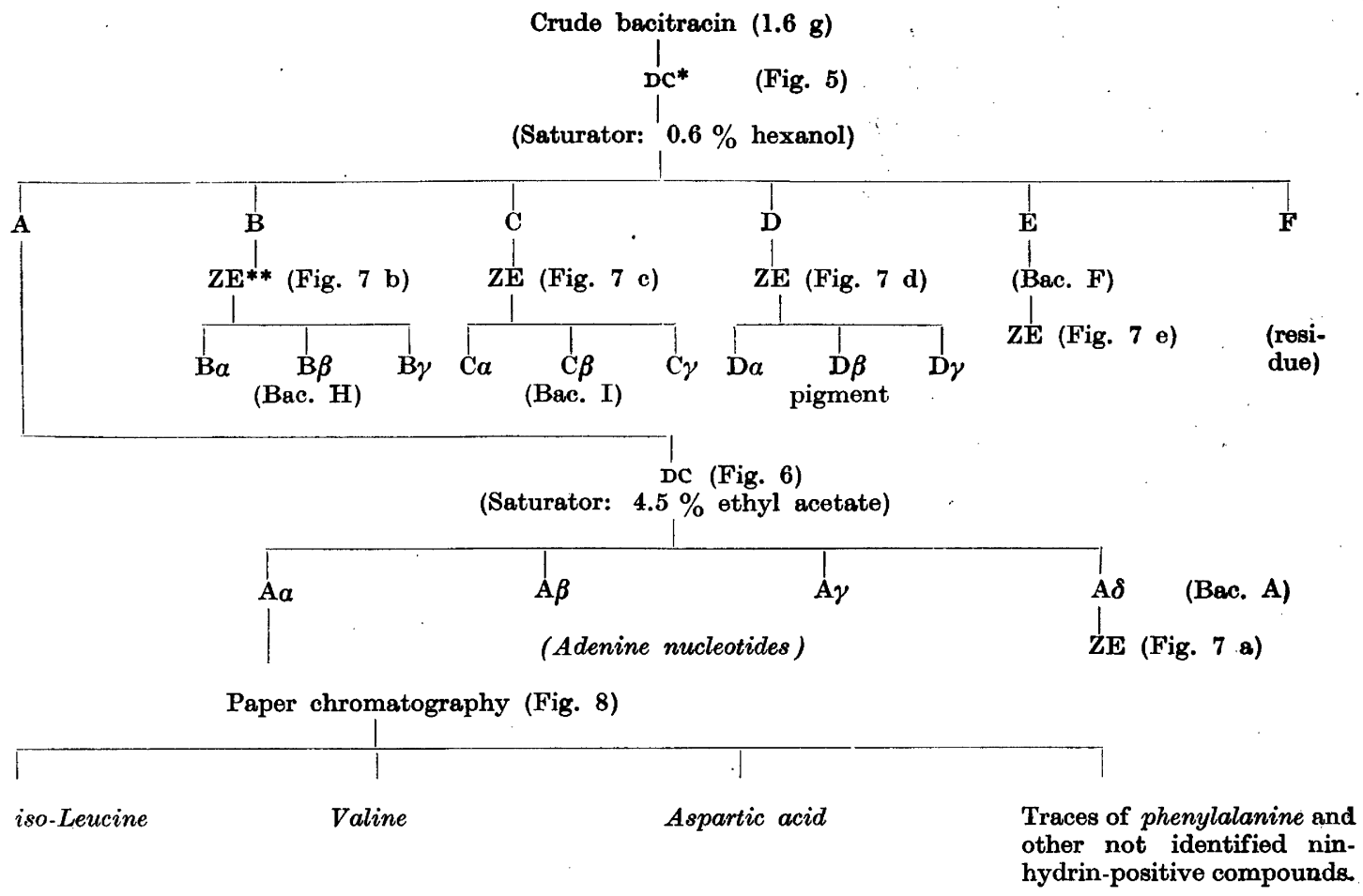

* $\quad \mathrm{DC}=$ Displacement chromatography on charcoal.

** $\mathrm{ZE}=\mathrm{Zone}$ electrophoresis in acetate buffer (pH 4.5; $\mu$ 0.05)

The investigation was started with a frontal analysis. This gave useful information about the complexity of the crude bacitracin sample. The fractionation on a preparative scale was then made by adsorption analysis on charcoal and zone electrophoresis, as summarized in the fractionation scheme above.

\section{EXPERIMENTAL TECHNIQUE}

Charcoal chromatography

Apparatus. Sectioned columns were used. These were of the type developed by Hagdahl ${ }^{10}$ but plastic-lined as described by $\mathrm{Li}$ and the author ${ }^{6}$. It is desirable to avoid contact of the solvent with metals, for traces of certain metal ions inactivate bacitracin 4.

Solvents. All solvents were made $0.1 \mathrm{~N}$ with acetic acid, because bacitracin $\mathrm{A}$ is reasonably stable in slightly acid solutions. Hexanol or ethyl acetate were found to be excellent saturators and the latter was also used as a displacer. These substances are easily removed before lyophilization by extraction with pentane or peroxide-free ether.

Adsorbents. Carbo Active (Merck, Darmstadt) was used in all experiments. Prior to use it was washed with $20 \%$ acetic acid. The charcoal was mixed with three times its weight of Hyflo Supercel or Celite. The columns were packed with this mixture when unsaturated charcoal or charcoal saturated with a solution of ethyl acetate was to be used. Saturation of the charcoal with hexanol could be carried out on the column, but

Acta Chem. Scand. 8 (1954) No. 10 

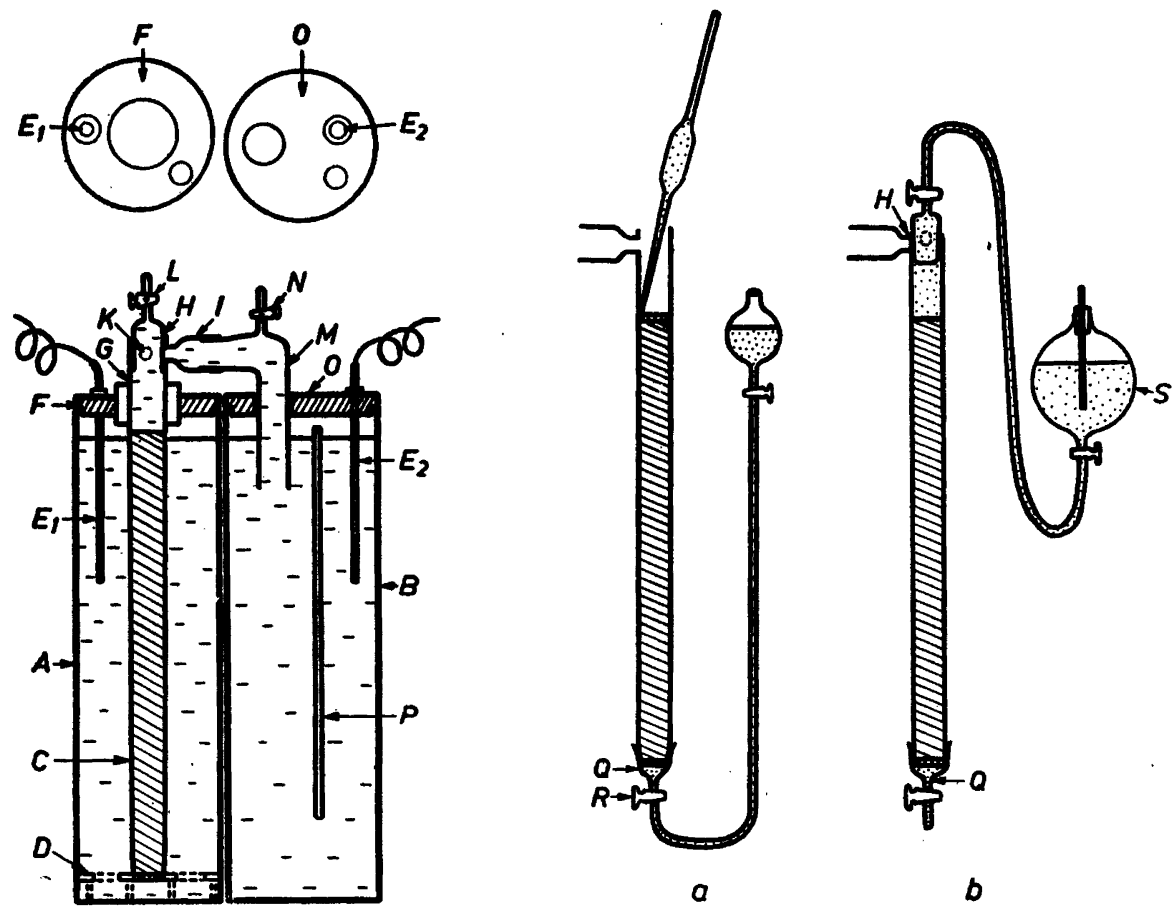

Fig. 1. Schematical drawing of the zoneelectrophoresis assembly with the two cylinder lids. Explanation of the letters in the text.

Fig. 2. a. Arrangement for application of the solution to be analyzed. $b$. Arrangement for displacement of the zones after the run.

in order to save time a combination of batchwise and column procedures was preferred. The charcoal-filter aid mixture $(200 \mathrm{~g})$ was suspended in $1000 \mathrm{ml} 0.6 \%$ hexanol solution and stirred for $1 / 2$ hour. The suspension was allowed to settle and the supernatant decanted and discarded. This treatment was repeated twice. To ensure satisfactory saturation the column of packed charcoal was always washed with the saturator solvent (at least three times the dead volume) before use. When ethyl acetate was used as a saturator, the column of untreated charcoal was saturated by washing with ethyl acetate solution until the ester appeared in the eluate.

Oxidation has never been found to occur on strongly pretreated charcoal.

\section{Zone electrophoresis}

Apparatus. A column ( $C$ in Fig. 1), similar to that described by Haglund and Tiselius ${ }^{12}$ and Flodin and Porath ${ }^{13}$, was used. It is made of glass - or quartz, if UV-absorption is to be recorded before elution. The solution is slowly forced into the column by about $5 \mathrm{~cm}$ water pressure (Fig. $2 \mathrm{a}$ ). The rinsing with buffer is also done cautiously. The zone is removed from the upper surface by lowering the level in the container. The flow should not exceed $10 \mathrm{ml}$ per hour. When the zone is in the desired position the stop-cock $R$ is closed and the column filled with buffer solution to the ground joint at the top. A connect- 
ing tube $M$, bent as a knee, and a top piece $H$ with a narrow tip and a stopcock $L$, are attached to the column. The ground joint of the top piece has an opening $K$, which is turned to coincide with the opening at the side $\operatorname{arm} I$. The stopcock $L$ is opened and $M$ turned upwards. Buffer solution is poured into $\mathbf{M}$ until the level reaches $L$. The stop-cock $L$ is now closed $H$ turned, $M$ emptied and turned downwards. The adapter $Q$ at the lower end of the electrophoresis column can then be detached without allowing the buffer solution to flow through the column. The column and the connecting tube can now be placed in the electrode vessels.

Two glass cylinders $A$ and $B$, each containing 7-8 liters of buffer solution, serve as electrode vessels (Fig. 1). Platinum wires twisted around rods of plastic are used as electrodes. The column $C$ is placed in the center of $A$. The lower end of the column is supported by a plastic disc of the same diameter as that of the cylinder $A$. The disc, which rests on $3 \mathrm{~cm}$ high rods of the same material, has a large hole in the center. This fits to the lower end of the column while other large holes allow passage of the current. The upper end of $C$ is kept in position by a stopper $G$ in the center of a plastic lid $F$ on the cylinder $A$. The lid $O$ of the cylinder $B$ has an opening for the tube $M$. Electrode rods $E_{1}$ and $E_{2}$ are inserted in the lids which also have openings for the gases formed by the electrode reactions. The electrode vessel $B$ also has a loose-fitting dividing wall of plastic $P$, to prevent electrode products from reaching $M$.

Liquid connection is simply effected by suction at the tip above $N$, subsequent closing of $N$ when $M$ is filled, and turning $K$ to open position. A siphon is placed between the two electrode vessels through the holes for gas outlet in the lids $F$ and $O$. When the buffer solutions in the two vessles are at the same level the siphon is removed and the electrodes connected to a power supply.

When a run is finished, $K$ is closed and the column and connecting tube $M$ are lifted off the electrode vessels. The tube $M$ is removed and the adapter $Q$ attached to the column. This is placed above a fraction collector and the flow rate is controlled by a siphon and a Mariotte flask $S$ as shown in Fig. 2 b.

The apparatus has the advantage of being easily manipulated. The large volume of buffer solution makes it possible to perform runs of long duration or several short runs without changing the buffer.

No extra cooling device is necessary especially not if the run is performed in a cold room.

General analytical methods

The material distribution was determined by the ninhydrin reaction and UV-absorption. The former was performed according to the description of Moore and Stein ", the latter by aid of a Beckman quartz-spectrophotometer model DU. The entire samples or suitable aliquots were taken for analysis.

\section{PRELIMINARY EXPERIMENTS}

Starting material. The main experiments and most of the other experiments were performed with a preparation (Bacitracin 50R 5 986), kindly supplied by Merck and Co., Inc., Rahway, N.J., U.S.A. It originated from Commercial Solvents Corporation (Lot No. B. 50070 605). At the time of this investigation the activity was found to be about 35 units/mg. The dry preparation was faintly yellowish while concentrated solutions were of a dark-brown color. 


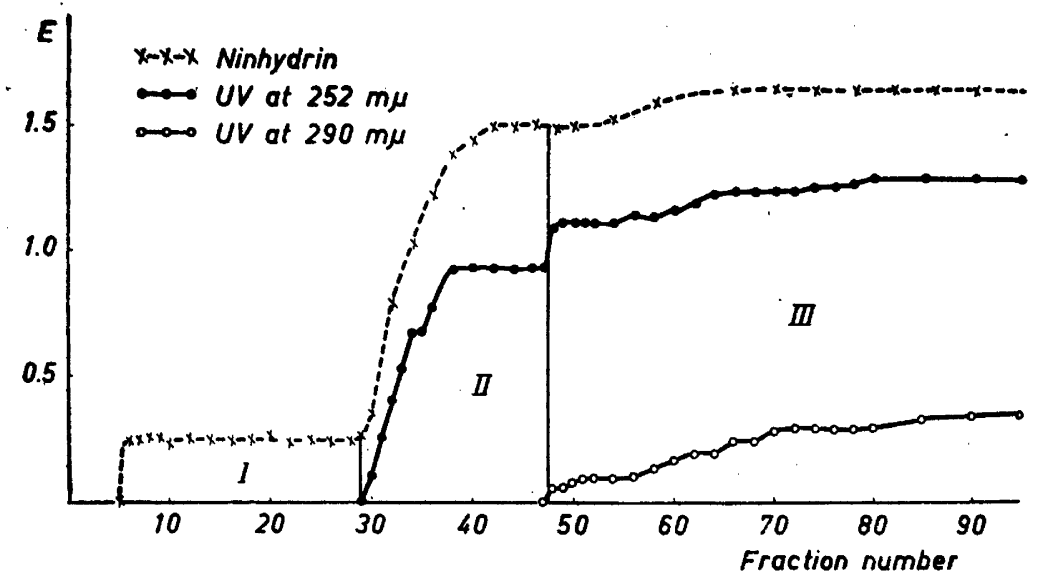

Fig. 3. Frontal analysis of crude bacitracin.

Crude bacitracin shows a very strong absorption in the ultraviolet region below 300 $m \mu$. Craig and Newton and their collaborators have found one (or possibly several) of the bacitracin polypeptides, bacitracin $F$, to have an absorption maximum at $290 \mathrm{~m} \mu$, while the most active component, bacitracin A, exhibits a slight maximum at about $252 \mathrm{~m} \mu$.

Frontal analysis of crude bacitracin. For orientation, a frontal analysis was carried out. The column consisted of three sections, 3.1, 1.6, and $0.8 \mathrm{ml}$, filled with a suspension of a mixture of charcoal and filter aid (proportions 1:3) in $0.1 \mathrm{~N}$ acetic acid. A solution of $0.15 \%$ crude bacitracin in $0.1 \mathrm{~N}$ acetic acid was pressed into the column at a rate of $6 \mathrm{ml}$ per hour. Fractions of $1.7 \mathrm{ml}$ were collected and measured spectrophotometrically at 252 and $290 \mathrm{~m} \mu$ after dilution to double the volume. For ninhydrin determinations $0.5 \mathrm{ml}$ of each fraction was taken.

The diagram (Fig. 3) reveals a variety of compounds of very different absorption and adsorption characteristics. Three regions can be distinguished. Region I, consisting of the least adsorbable material, gives a strong ninhydrin color but no significant absorption at the wavelength measured. No antibiotic activity with the Corynebacterium xerosis test was found in this region. Paper chromatography of the material in this fraction gave diagrams identical with those of $\mathrm{A} \alpha$ (see Fig. 8 and p. 1821). It consists chiefly of amino acids. In Region II a rise in the absorption at $252 \mathrm{~m} \mu$ appears in fraction 30. That the additional material emerging in this region is heterogeneous can be seen from the change in the intensity of the ninhydrin color/UV-absorption ratio of fractions 30 to 42 . The first step of the $252 \mathrm{~m} \mu$-curve obtained in several experiments, points to the same conclusion. The biological activity roughly parallels the UV-curve. In tube No. 48 another rise in the $252 \mathrm{~m} \mu$ curve occurs and at the same time material absorbing at $290 \mathrm{~m} \mu$ emerges from the column. All the curves show a small increase in absorption in Region III. This strongly indicates the appearance of several other compounds. The breakthrough of a yellow pigment is also observed in this region.

The results of the frontal analysis suggest excellent possibilities for an efficient fractionation by charcoal chromatography. 

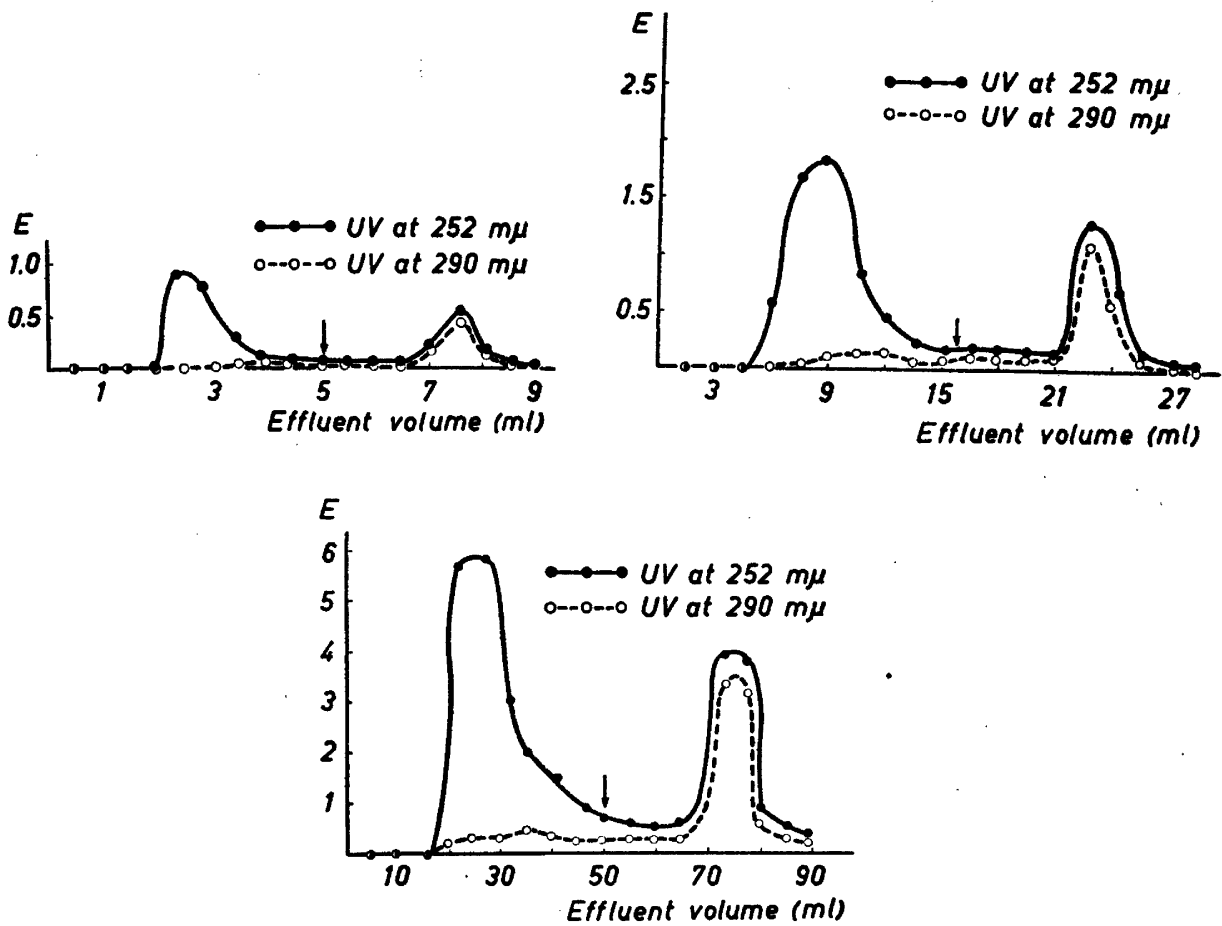

Fig. 4. Displacement chromatograms of crude bacitracin on pretreated charcoal columns. Saturated ethyl-acetate was introduced at the top of the column at effluent volumes indicated by the arrows. a. A $5 \mathrm{mg}$ sample applied to a column of $1.6 \mathrm{ml}$ volume. $b . A 25 \mathrm{mg}$ sample applied to a $4.7 \mathrm{ml}$ column. c. $A 125 \mathrm{mg}$ sample applied to a $18.8 \mathrm{ml}$ column.

Displacement analysis of crude bacitracin. Frontal analysis of crude material revealed that the yellow pigment and the material absorbing strongly at $290 \mathrm{~m} \mu$ were the components most strongly adsorbed on charcoal. Thus, the charcoal must be pretreated with a saturator strong enough to prevent extensive irreversible adsorption of material absorbing at $290 \mathrm{~m} \mu$. Preliminary displacement analysis experiments were carried out to determine suitable working conditions.

In the first series the column volume $(1.6 \mathrm{ml})$, the charge $(5 \mathrm{mg})$ and the flow rate $(2 \mathrm{ml}$ per hour) were most the same, while the concentration of the flowing saturator ( $n$-hexanol in $0.1 N$ acetic acid) was varied. The hexanol-acetic acid solvent saturated with ethyl acetate was used as a displacer. A $0.6 \%$ hexanol solution was chosen for further investigation, because in this case a complete recovery within experimental error, was achieved. Fractionation was also satisfactory (Fig. 4 a).

In a new series, the charge was raised to $25 \mathrm{mg}$ and the flow rate to $5 \mathrm{ml}$ per hour. The amount of adsorbent was varied. The use of a $4.7 \mathrm{ml}$ column volume resulted in about $95 \%$ recovery of material absorbing at $290 \mathrm{~m} \mu$ (Fig. 4 b).

Satisfactory fractionation and recovery were obtained when $125 \mathrm{mg}$ of material were placed on a column of $18.8 \mathrm{ml}$ volume (Fig. $4 \mathrm{c}$ ). In this case as in all large scale experiments, the flow rate was $20 \mathrm{ml}$ per hour. Approximately the same charge/column volume ratio was used when gram quantities were fractionated.

Acta Chem. Scand. 8 (1954) No. 10 


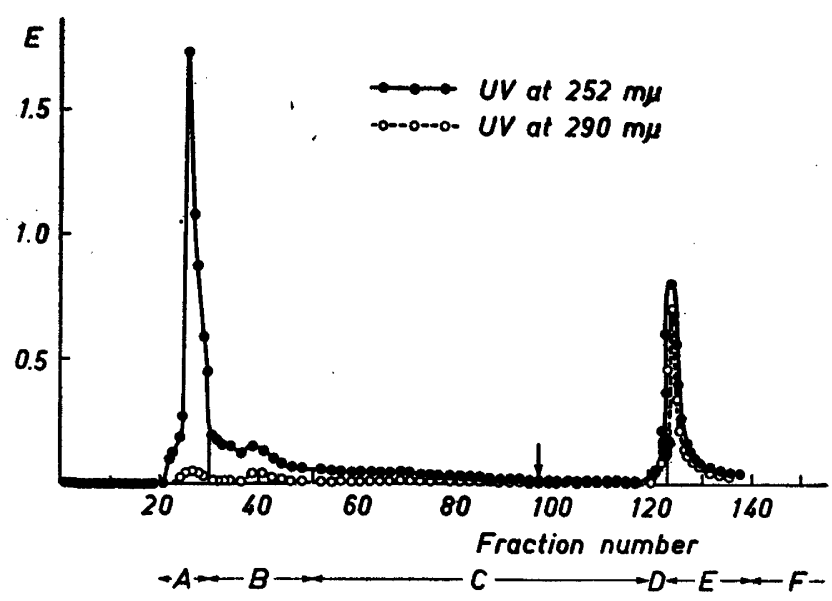

Fig. 5. Diagram showing the fractionation of $1.6 \mathrm{~g}$ crude bacitracin on a column made of seven $31.4 \mathrm{ml}$ sections. The small fractions were pooled to the larger Fractions $A-F$ as indicated. The arrow has the same meaning as in Fig. 4.

\section{PREPARATIVE FRACTIONATIONS}

\section{Charcoal chromatography}

Starting material: See "Preliminary Experiments".

Displacement chromatography of crude bacitracin. A column of seven $31.4 \mathrm{ml}$ sections filled with charcoal saturated with $0.6 \%$ hexanol in $0.1 \mathrm{~N}$ acetic acid was loaded with $1.6 \mathrm{~g}$ crude bacitracin dissolved in $4 \mathrm{ml}$ of the same solvent. The flow rate was adjusted to $20 \mathrm{ml}$ per hour by applying a pressure of about $3 \mathrm{~kg}$ per $\mathrm{cm}^{2}$. Fractions were collected in a tima-controlled fraction collector $(10 \mathrm{~min}$. per fraction). When $400 \mathrm{ml}$ solvent had passed through the column the elution was stopped and the displacement was started, keeping the flow at the same rate as before. The distribution of material with respect to UV-absorption at 252 and $290 \mathrm{~m} \mu$ was determined and the small fractions were pooled into six large fractions, as indicated in the diagram (Fig. 5). The solutions were extracted with peroxide-free ether to remove hexanol and ethyl acetate. After evaporation of the excess ether the solutions were lyophilized.

All the freeze-dried samples were non-hygroscopic powders. Fraction D, which contained the pigment, was yellowish; the others were white. Fraction A possessed the bulk of the activity, while Fraction F was least potent. The material was distributed as follows: Fraction A, $741 \mathrm{mg}$; B, $112 \mathrm{mg}$; C, $129 \mathrm{mg}$; $\mathrm{D}, 117 \mathrm{mg}$; E, $325 \mathrm{mg} ; \mathrm{F}, 36 \mathrm{mg}$. Thus $92 \%$ was recovered on a weight basis.

Displacement chromatography of Fraction A. Some of the material of Fraction $A$ is strongly adsorbed on untreated charcoal. Since the material of Fraction A could pass the column in the experiment described above without being adsorbed a suitable saturator concentration of hexanol could presumably be found. However, ethyl acetate was used instead, because much experience had been gained from other preliminary experiments using this substance as a saturator. Acceptable yields and fractionations were obtained with $4-4.5 \%$ ethyl acetate. Large scale experiments were performed as follows. 


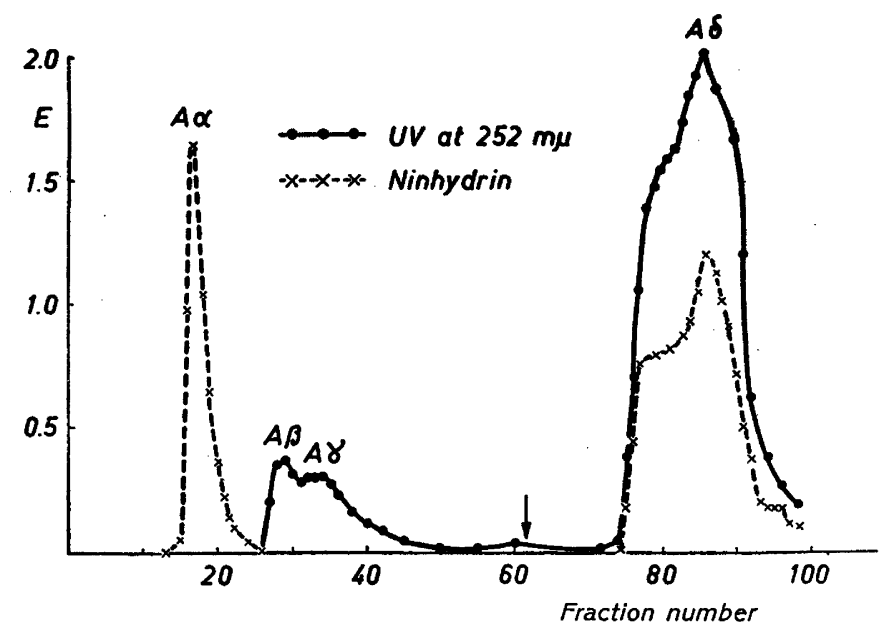

Fig. 6. Displacement analysis diagram of $512 \mathrm{mg}$ of Fraction A (see Fig. 5) obtained from a chromatographic run on charcoal pretreated with $4.5 \%$ ethyl-acetate. $A$ column of four $31.4 \mathrm{ml}$ sections was used. Displacement was started when $300 \mathrm{ml}$ of eluent had passed the column.

A solution of Fraction A containing $0.6 \%$ hexanol was freed from the latter by extraction with pentane and evaporation of the excess hydrocarbon in vacuo. The hexanol-free solution so obtained $(15 \mathrm{ml})$, containing $512 \mathrm{mg}$ of solid, was pressed into a column of four $31.4 \mathrm{ml}$ sections containing the adsorbent in equilibrium with $4.5 \%$ ethyl acetate in $0.1 N$ acetic acid. Elution was effected with $4.5 \%$ ethyl acetate. When $300 \mathrm{ml}$ had passed the column the displacement with saturated ethyl acetate was started. Fractions of $5 \mathrm{ml}$ volume were collected. The material containing fractions were pooled, the ethyl acetate removed and the solutions lyophilized.

The elution peak $\mathrm{A} \alpha$ (Fig. 6) contained $13 \mathrm{mg}$ of ninhydrin reactive material which showed very slight absorption at $252 \mathrm{~m} \mu$. The material in this fraction is, of course, the same as that in the first step of the frontal analysis chromatogram (Region I). From $\mathrm{A} \beta$ and $\mathrm{A} \gamma$ only about $2 \mathrm{mg}$ were recovered, in spite of the strong absorption. From A $\delta 442 \mathrm{mg}$ were recovered.

\section{Zone electrophoresis of various fractions}

Fraction $A \delta$. The column $(45 \times 3.5 \mathrm{~cm})$ was filled with a suspension of cellulose powder in sodium acetate buffer of $\mathrm{pH} 4.5$ and $\mu 0.05$. The packing was made with a hydrostatic pressure of about two meters of water ${ }^{15}$. Forty-five milligrams of material were dissolved in $1 \mathrm{ml}$ of buffer solution and pressed into the column ${ }^{13}$. The zone was first displaced to $8 \mathrm{~cm}$ below the surface of the column using $50 \mathrm{ml}$ of buffer solution. A current of $25 \mathrm{~mA}$ was passed through the column for 55 hours $(400 \mathrm{~V}$ between the electrodes). After disconnecting the tube, the buffer containing the peptide material was displaced from the column at a rate of $25 \mathrm{ml}$ per hour and collected in $5 \mathrm{ml}$ portions.

Under these conditions (Fig. 7 a) Fraction A $\delta$ appears to be electrophoretically homogeneous. Paper electrophoresis at $\mathrm{pH} 5.3$ and 6.0 give a single spot. 

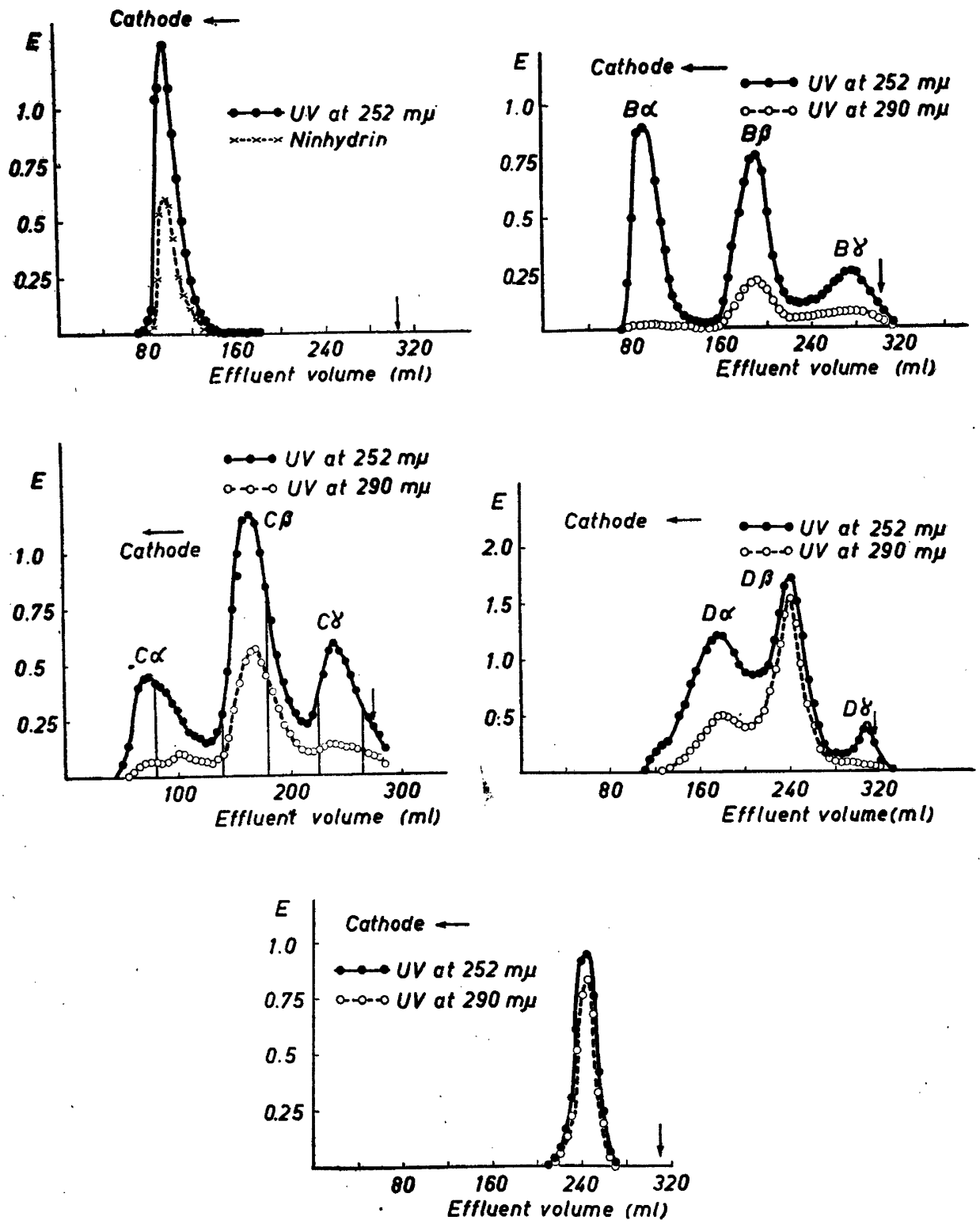

Fig. 7. Zone electrophoresis of fractions obtained from charcoal chromatograms (see Figs. 5 and 6 ). $a$. Fraction $A \delta$. b. Fraction $B$. c. Fraction $C$. d. Fraction $D$. e. Fraction $E$. The arrows indicate the volume in the column between the filter plate and the starting position of the original zone. All runs were carried out with acetate buffer $(p H 4.5 ; \mu 0.05)$. For details see text. 
Other experiments, performed in a similar way, are compiled in Table 1.

Table 1.

\begin{tabular}{ccccccc}
\hline Fraction & $\begin{array}{c}\text { Amount } \\
\text { applied } \\
(\mathrm{mg})\end{array}$ & $\begin{array}{c}\text { Column } \\
\text { (length } \times \\
\text { diameter) } \\
(\mathrm{cm})\end{array}$ & $\begin{array}{c}\text { Distance of } \\
\text { starting zone } \\
\text { from the } \\
\text { upper surface } \\
\text { (cm) }\end{array}$ & $\begin{array}{c}\text { Current } \\
(\mathrm{mA})\end{array}$ & $\begin{array}{c}\text { Duration } \\
\text { (hours) }\end{array}$ & $\begin{array}{c}\text { Diagram } \\
\text { in } \\
\text { Fig. }\end{array}$ \\
\hline $\mathrm{B}$ & 50 & $45 \times 3.5$ & 6 & 28 & 60 & $7 \mathrm{~b}$ \\
\hline $\mathrm{C}$ & 80 & $40 \times 3.5$ & 5 & 20 & 70 & $7 \mathrm{c}$ \\
\hline $\mathrm{D}$ & 50 & $45 \times 3.5$ & 8 & 28 & 60 & $7 \mathrm{~d}$ \\
\hline $\mathrm{E}$ & 50 & $45 \times 3.5$ & 6 & 28 & 60 & $7 \mathrm{e}$ \\
\hline
\end{tabular}

\section{DISCUSSION}

The completely inactive Fraction A $\alpha$ (Fig. 5) was further analysed by paper chromatography. Hydrolysis changes the chromatogram only slightly with respect to ninhydrin-positive substances (Fig. 8). The strongest spot appears in the same region as leucine and isoleucine while a weaker spot occupies the position of valine. Besides these amino acids, aspartic acid and traces of phenylalanine, as well as a number of other unidentified ninhydrin-positive compounds are present. The large quantity of isoleucine is noteworthy for isoleucine has been reported as a DNP-derivative in the hydrolysate of dinitrofluorobenzene-treated bacitracin $\mathbf{A}^{\mathbf{1 6}}$. All commercial bacitracin samples

Fig. 8. Paper chromatograms of unhydrolyzed and hydrolyzed fraction $A \alpha$, and mixtures of glycine, valine and is o leucine. Solvent system: butanolacetic acid-water $(4: 1: 5)$.

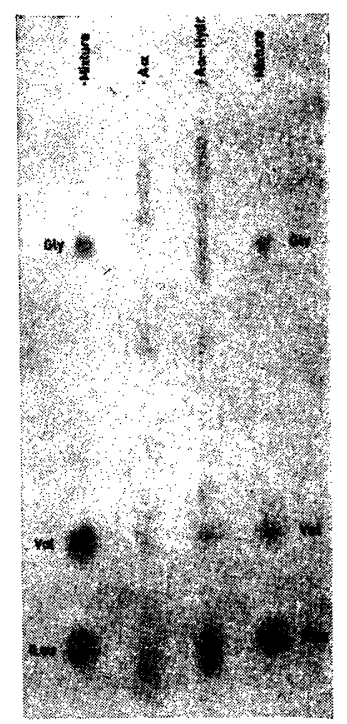

Acta Chem. Scand. 8 (1954) No. 10 


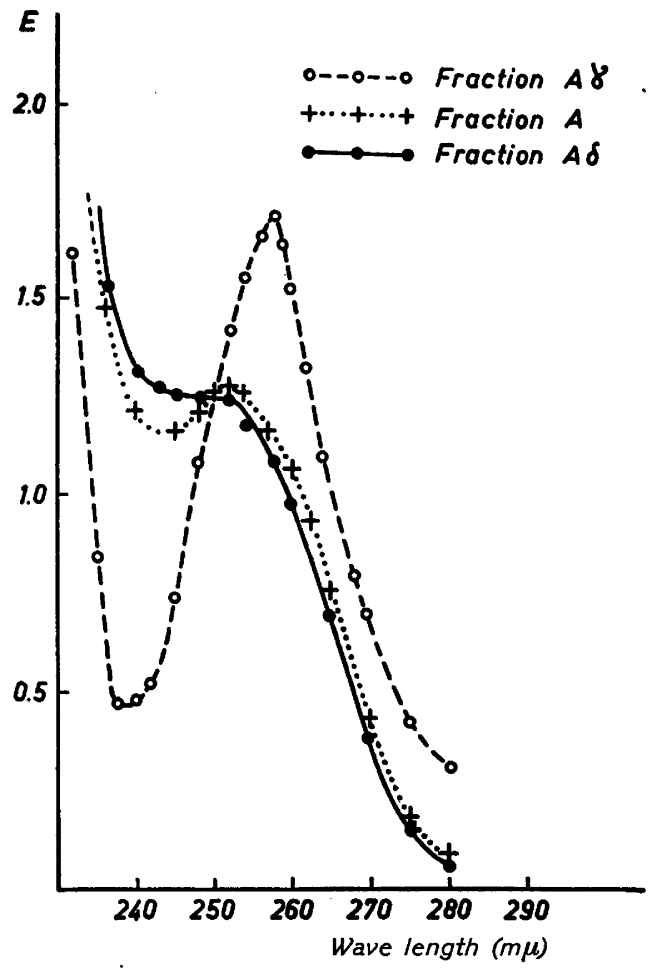

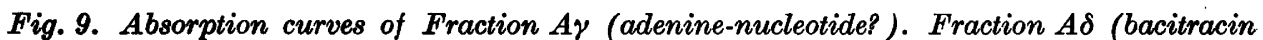
$A$ ) and Fraction $A$ (bacitracin $A$ contaminated by $A \beta$ and $A \gamma$ ). Ordinate: Optical density for $0.1 \%$ solutions in dilute acetic acid ( $p H$ 3.0).

analyzed by the present author were contaminated with the same amino acids, although in different amounts. These amino acids have not been accounted for in fractionations, reported in literature, where counter-current distribution was used $^{1-4}$. The yield of $A \alpha$ material in a number of crude bacitracin samples varies between 1 and $3 \%$. The small amount of DNP-iso-leucine found in bacitracin A purified with counter-current distribution may possibly originate not from bacitracin A itself but from small amounts of contaminating free isoleucine ${ }^{2}$. Under the conditions used here zone electrophoresis does not remove isoleucine from bacitracin A (Fraction A). Since bacitracin A is fairly unstable, it might be thought that some chemical reaction which releases free isoleucine occurs on the charcoal. Rechromatographing Fraction A $\delta$ on a charcoal column under the same conditions used for the purification proved that bacitracin A does not change its properties on the column. Only a single displacement peak was obtained and paper chromatography definitively proved the absence of any free amino acid. The difficulties in the separation are probably to be explained by an association of the free amino acids with the peptides.

Adsorption chromatography appears to be a powerful method of splitting such complexes. 


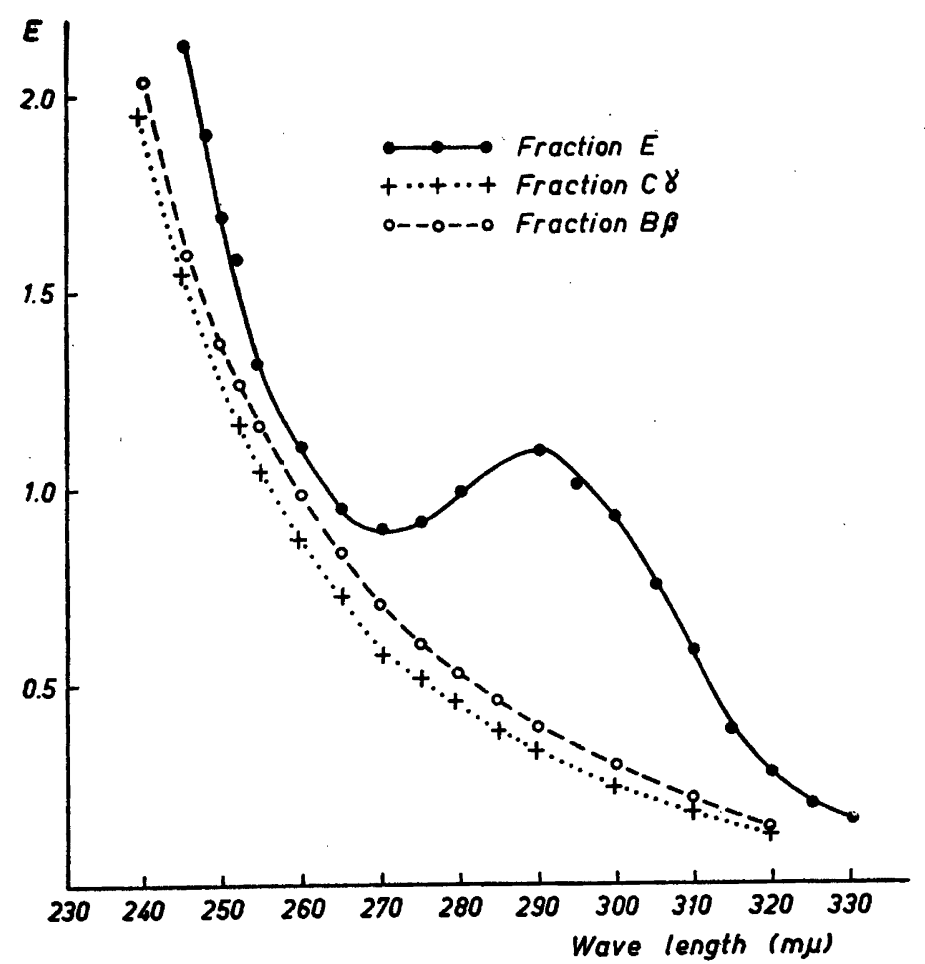

Fig. 10. Absorption curves of Fraction $B \beta$ (bacitracin $H$ ) and Fraction $C \gamma$ (bacitracin $I$ ) and Fraction $E$ (bacitracin $F$ ). Ordinate: Optical density for $0.1 \%$ solutions in dilute acetic acid ( $p H$ 3.0).

The fractions $A \beta$ and $A \gamma$ show ultraviolet absorption curves in solutions of different $\mathrm{pH}$ which are similar to adenine and adenine nucleotides (Fig. 9). Due to lack of material a closer examination of these fractions could not be undertaken. Chromatography of $\mathrm{A} \beta$ and $\mathrm{A} \gamma$. after heating to $120^{\circ} \mathrm{C}$ with $6 \mathrm{~N}$ hydrochloric acid in sealed tubes for 20 hours did not reveal any significant amount of ninhydrin-positive substances. This indicates that adenine, if present, is in a bound state. Fraction A $\gamma$ mixed with $A \delta$ in certain proportions give absorption curves similar to those of bacitracins $\mathrm{C}$ and G. ${ }^{4}$ It is therefore questionable whether the latter compounds are pure bacitracin peptides.

The form of the peak A $\delta$ might give the impression of heterogeneity. However, the material in different parts of the peak gives identical absorption spectra (that of $\mathrm{A} \delta$ in Fig. 9). The antibacterial activity is also uniformly distributed. Most likely the asymmetry is caused by non-equilibrium conditions during the displacement. The high potency $(76 \mathrm{u} / \mathrm{mg})$ and chemical properties of fraction A proves that it consists of bacitracin A. The absorption curve of Fraction $A$ is very similar to the bacitracin B curve of Craig et al. and has a slight maximum at $252 \mathrm{~m} \mu$. After removing the $\mathrm{A} \beta$ and $A \gamma$ substances the curve becomes similar to that of bacitracin $\mathrm{A}$. The differences in absorption 
curves of the bacitracins $A$ and $B$ found by Craig and his collaborators 1 may be explained by traces of contaminating adenine-compounds.

The Fractions B, C, and D contain a number of different components, two of which, $\mathrm{B} \beta$ and $\mathrm{C} \gamma$, have been more closely examined. With the exception of $\mathrm{B} \alpha$, the other fractions are certainly not pure. $\mathrm{B} \alpha$ consists of bacitracin $A$. Fractions $\mathrm{B} \beta$ and $\mathrm{C} \gamma$ consist of peptides structurally related to bacitracin $A$ as will be shown in a forthcoming paper. The absorption curves (Fig. 10) differ from those of the reported bacitracins. Since these fractions cannot be further purified by these methods the peptides in Fractions $\mathrm{B} \beta$ and $\mathrm{C} \gamma$ will be referred to as bacitracin $H$ and bacitracin $I$, respectively.

Fraction E seems to be electrophoretically homogeneous. The substance in this fraction has an absorption curve similar to or identical with that reported for bacitracin $\mathrm{F}$.

From this investigation, it appears that fractionation of bacitracin polypeptide mixtures by zone electrophoresis and charcoal chromatography are capable of considerable further purification than is readily effected using counter-current distribution alone. It should be borne in mind that extremely small amounts of purine material would considerably change the absorption curve in the range $250-260 \mathrm{~m} \mu$. The bacitracins reported in the literature may, therefore, be pure enough for further chemical investigations.

Acknowledgements. The author wishes to express his gratitude to Professor A. Tiselius and to Dr. B. Drake for helpful and stimulating discussions. He is indebted to Mr. S. Jerstedt for able technical assistance and to Dr. L. Kavanau for linguistic revision of the manuscript. Thanks are also due to Merck and Co. Inc., Rahway, N.J., U.S.A. for the supply of commercial bacitracin.

This work has been supported by grants from the Swedish Natural Science Research Council, The Rockefeller Foundation and The Wallenberg Foundation.

Added in proof: Recent studies by the present author on the structure of bacitracin $A$ and $\mathbf{F}$ as obtained by the procedures described here are in agreement with the amino acid sequence published by Craig, Hausmann and Weisiger (J. Am. Chem. Soc. 76 (1954) 2839) and that found by Lockhart and Abraham (personal communication). There is thus no reason to believe that the compounds here isolated are different from those described by these authors.

\section{REFERENCES}

1. Craig, L. C., Ǵregory, J. D. and Barry, G. T. J. Clin. Invest. 24 (1949) 1014.

2. Craig, L. C., Weisiger, J. R., Hausmann, W. and Harfenist, E. J. J. Biol. Chem. 199 (1952) 259.

3. Newton, G. G. F. and Abraham, E. P. Biochem. J. (London) 47 (1950) 257.

4. Newton, G. G. F. and Abraham, E. P. Biochem. J. (London) 53 (1953) 597.

5. Porath, J. Acta Chem. Scand. 6 (1952) 1297.

6. Porath, J. and Li, C. H. Biochim. et Biophys. Acta 13 (1954) 268.

7. Tiselius, A. and Hahn, L. Kolloid.-Z. 105 (1943) 177.

8. Singe, R. L. M. and Tiselius, A. Acta Chem. Scand. 3 (1949) 231.

9. Drake, B. Arkiv Kemi 7 (1954). In press.

10. Hagdahl, L. Acta Chem. Scand. 2 (1948) 574.

11. Arriagada, A., Savage, M. G., Abraham, E. P., Heatley, H. G. and Sharp, A. E. Brit. J. Exptl. Pathol. 30 (1949) 425.

12. Haglund, H. and Tiselius, A. Acta Chem. Scand. 4 (1950) 957.

13. Flodin, P. and Porath, J. Biochim. et Biophys. Acta 13 (1954) 175.

14. Moore, S. and Stein, W. H. J. Biol. Chem. 176 (1948) 367.

15. Flodin, P. Private communication.

16. Lockhart, I. M., Newton, G. G. F., and Abraham, E. P. Nature 173 (1954) 536.

Received July 16, 1954.

Acta Chem. Scand. 8 (1954) No. 10 